\title{
Protective Measures for Clinical Physicians When Performing Tracheal Intubation for Patients with Acute Respiratory Syn- drome of the Coronavirus Disease 2019 (COVID-19)
}

\author{
Xianzeng Chen, Weilan Wu and Xiaodan $\mathrm{Wu}^{*}$ \\ Department of Anesthesiology, Fujian Medical University, China
}

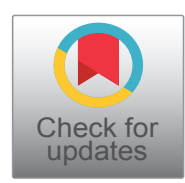

*Corresponding author: Xiaodan Wu, Department of Anesthesiology, The South Branch of Fujian Provincial Hospital, Institution of Clinical Medicine, Shengli Clinical Medical College, Fujian Medical University, No.516 the South of Jin-Rong Road, Cang-Shan District, Fuzhou, Fujian, 350028, China

\section{Introduction}

Since December 2019, the outbreaks of COVID-19 global pandemic has occurred, currently considered, first on Wuhan [1], and appeared successively on many other countries in China. As an acute respiratory infectious disease, it has been defined as a class $B$ infectious disease, according to the regulations of the People's Republic of China on the prevention and control of infectious diseases, and managed as a top class infectious disease.

For those with severe acute respiratory syndrome of coronavirus infection disease, tracheal intubation is the most commonly used procedure when establishing a temporary airway. Clinical intervention, such as endotracheal intubation or tracheotomy, sputum suction, and throat swab collection, is required close contact, and at considerable exposure risk.

As the virus is highly infectious, medical workers should master effective protective measures in advance to avoid infection [2].

\section{Objectives}

By observing the advanced interventions in the process of admitting and diagnosing patients in the comprehensive medical institutions, this study concluded that: Firstly, the arrangement of a professional team responsible for endotracheal intubation is preferable in such situations. Secondly, the positive pressure head shield can avoid directly contact of pathogens in the patient's exhaled air during intubation. Thirdly, when the supply of protective articles is in short supply [3] or in extreme conditions [4], alternative protective plans shall be adopted.

\section{Methods}

\section{Theoretical foundation}

The transmission of infectious diseases needs three elements are the source of infection, the route of transmission, and the susceptible population.

The following statements are based on the diagnosis and treatment program of novel coronavirus pneumonias disease (trial of the $7^{\text {th }}$ edition).

Source of infection: Novel coronavirus infection is confirmed, asymptomatic infection may also become a source of infection.

Route of transmission: Respiratory droplets and close contact are the main routes of transmission. It is possible to propagate through aerosols when exposed to high concentration aerosols for a long time in a relatively closed environment. Because novel coronavirus can be isolated from feces and urine, attention should be paid to the spread of aerosols or contacts caused by environmental pollution by feces and urine.

Susceptible population: The population is generally susceptible. 


\section{Protective measures}

Interventions against infectious sources: Isolate the source of infection and reduce exposure. For patients who do not need respiratory support, they should wear masks regularly. For critical patients who need respiratory support, protective measures are taken to reduce secretion splashing, droplets and aerosols during tracheal intubation. At present, for patients without mask ventilation difficulties and tracheal intubation difficulties, rapid induction anesthesia can be used to provide low-risk tracheal intubation operation conditions under deep sedation and with the use of muscle relaxants. It can significantly reduce the production of secretion splashes, droplets and aerosols, and reduce the risk of direct exposure to operators.

Standardization process: Referred to expert recommendation of novel coronavirus pneumonia (the $1^{\text {st }}$ edition):

\section{(1) Position selection}

The patient took the olfactory position.

The patients with obesity were in high head position or clivus position.

\section{(2) Pre-oxygenation}

Prepare face mask and simple respirator, cover the mouth and nose with double-layer wet saline gauze, and start 5 minutes of pre oxygenation (Figure 1).

For patients with noninvasive ventilation assisted breathing, prepare face mask and simple respirator, use $100 \%$ pure oxygen ventilation, and start 5 minutes of pre oxygenation.

\section{(3) Induction stage}

\section{Intravenous Midazolam 2-5 mg}

A small dose of Etomidate (patients with hemodynamic instability) or a small dose of Propofol (patients

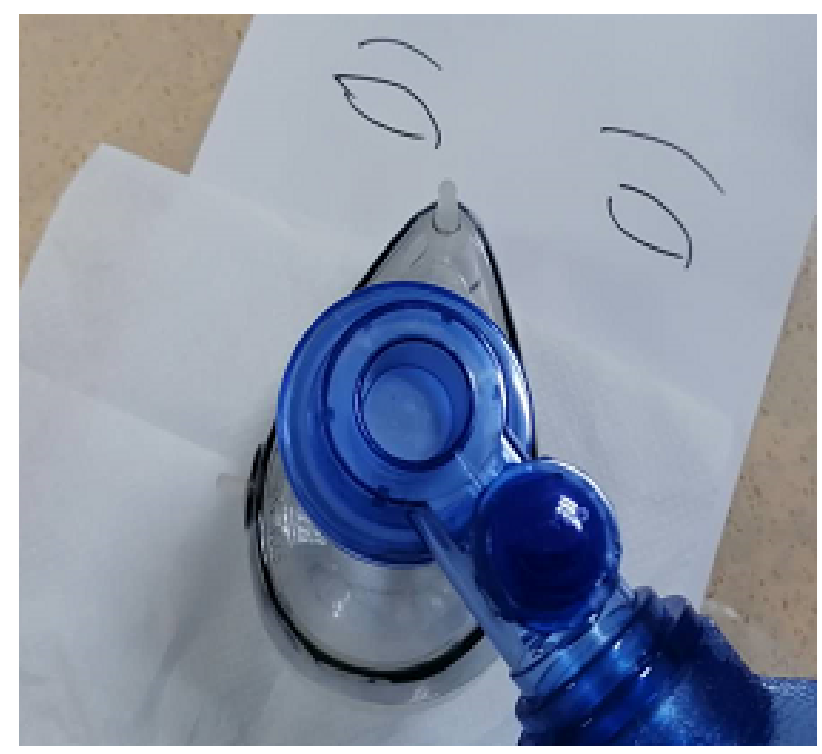

Figure 1: Double-layers saline gauze has covered the mouse and nose. with hemodynamic stability). Sufentanil or Remifentanil may be given as appropriate to reduce the stress reflex during tracheal intubation.

\section{(4) Operating conditions}

Rocuronium $0.9 \mathrm{mg} / \mathrm{kg}$ (the first choice) or Succinylcholine $1 \mathrm{mg} / \mathrm{kg}$ were injected intravenously and intubated one minute later.

Succinylcholine is forbidden in patients with hyperkalemia.

At present, Rocuronium also has antagonists, Sugammadex Sodium Injection.

In some patients with pulmonary hypertension, Rocuronium can show obvious side effects of pulmonary hypertension.

\section{(5) Full exposure}

Cricoid cartilage compression is helpful for glottic exposure and tracheal intubation, and can also reduce the occurrence of regurgitation and aspiration.

\section{(6) Intermittent oxygen supply}

If the patient can't tolerate the short-term non ventilation, the low tidal volume method should be selected when the mask ventilation is needed.

During the operation of endotracheal intubation, high flow oxygen was given to avoid aggravating hypoxemia.

\section{(7) Intubation supplies}

It is recommended to use disposable laryngoscope and visual laryngoscope for intubation.

When the intubation is difficult, the disposable visual tube core can be used to assist the intubation.

\section{(8) Suction device}

Closed airway suction is recommended to reduce open suction.

Deficiencies of the above processes: In order to avoid aerosol diffusion, step (2) use double-layer saline gauze to cover the mouth and nose slightly thin, increase the number of layers can provide better effect on preventing aerosol leakage, but at the same time, increase the difficulty of ventilation of the mask, of which hypoxemia is more likely to happen.

To improve the above interventions: Put the multi-layer saline gauze on the outside of the mask, and the covering area should be $5 \mathrm{~cm}$ beyond the edge of the mask. It not only meets the needs of filtering and preventing aerosol leakage, but also has no significant impact on ventilation effect.

Further improve the interventions: As the saline gauze placed on the outside of the mask prevent close contact with the patient's airway, gauze that damped with $70 \%$ 


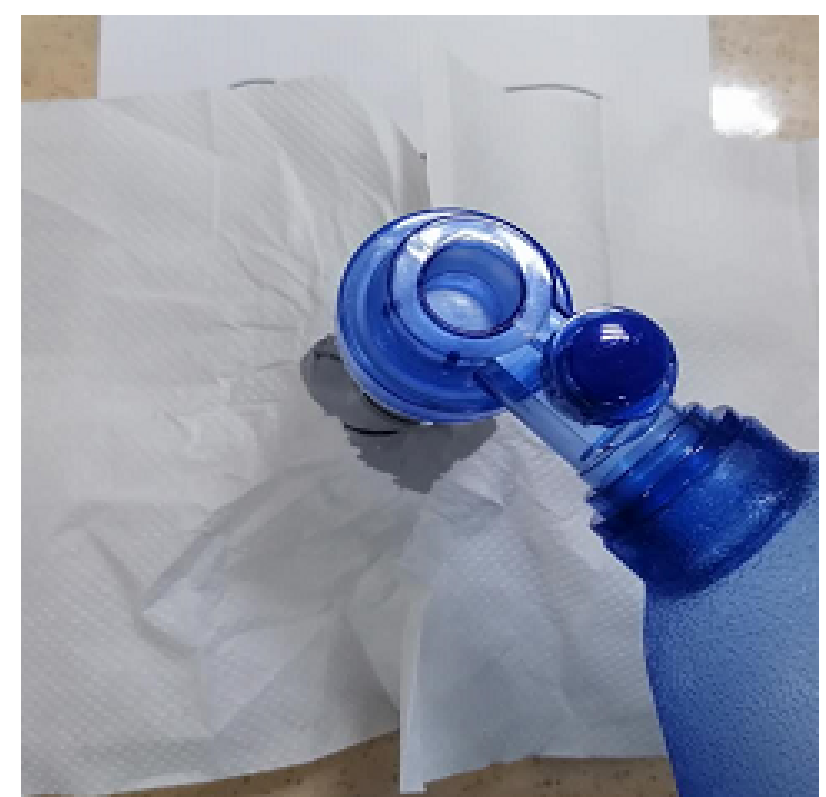

Figure 2: Multi-layers gauze damped with saline or $70 \%$ alcohol has covered the outer of the mask.

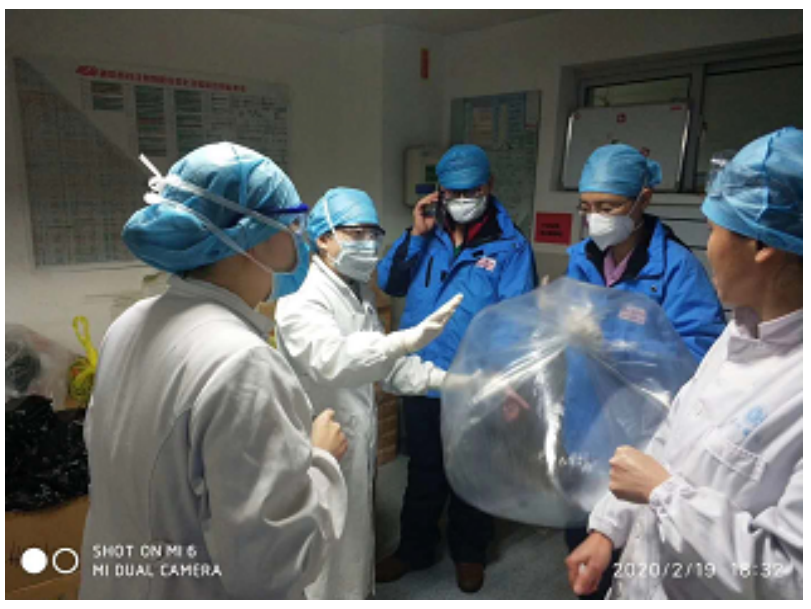

Figure 3: On hand materials in the ward (large transparent plastic bag).

alcohol is used instead, which has stronger isolation and protection effect, especially for patients who need to receive pressure ventilation (Figure 2).

\section{Interventions on transmission routes}

Large and transparent plastic shield: It is with applying the large and completely transparent plastic shield, which covered the upper part of head and body of the patient (normally of $70 \mathrm{~cm} \times 70 \mathrm{~cm}$ in size). High concentration oxygen flow supply by mask, pressure ventilation, endotracheal intubation, sputum suction, etc. can be carried out under the transparent plastic shield. By blocking the transmission route, this cheap plastic shield can be used to prevent accidentally contact of the splashed secretion, droplets, aerosols, etc., at the same time, reducing the contaminant of the environment, and greatly improving the safety of such procedures (Figure 3, Figure 4 and Figure 5).

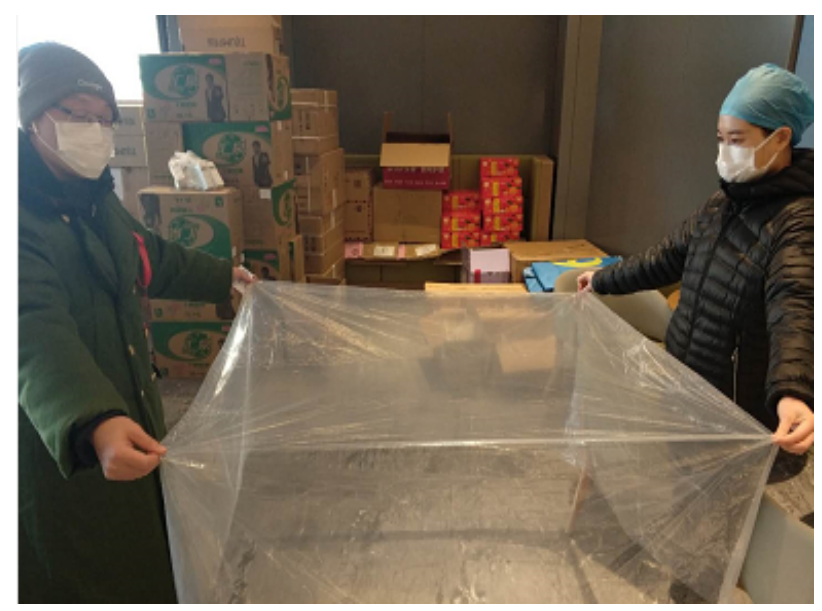

Figure 4: Customized large and transparent plastic shield.

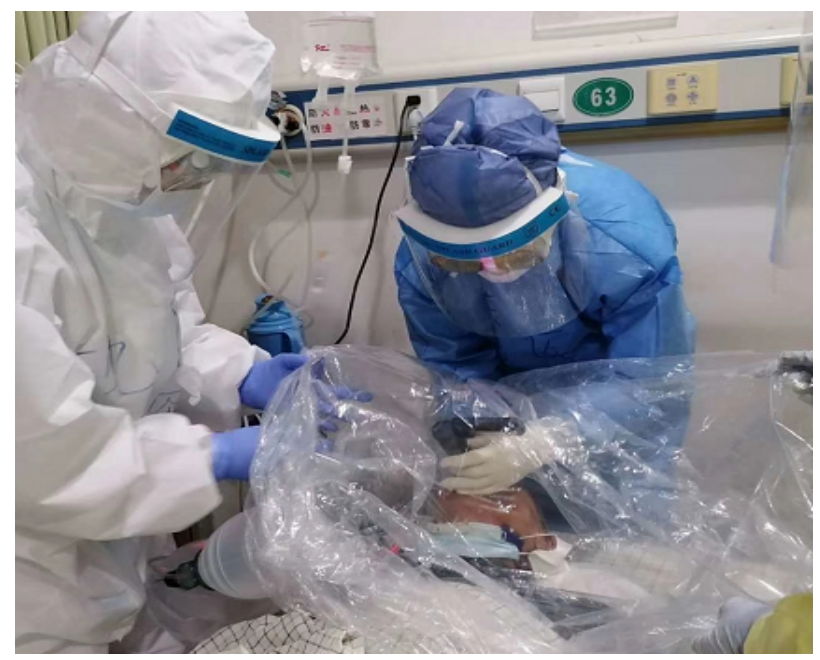

Figure 5: These pictures are captured on site when performing tracheal intubation, the same as pressure oxygen supply or sputum suction can be done under the plastic shield.

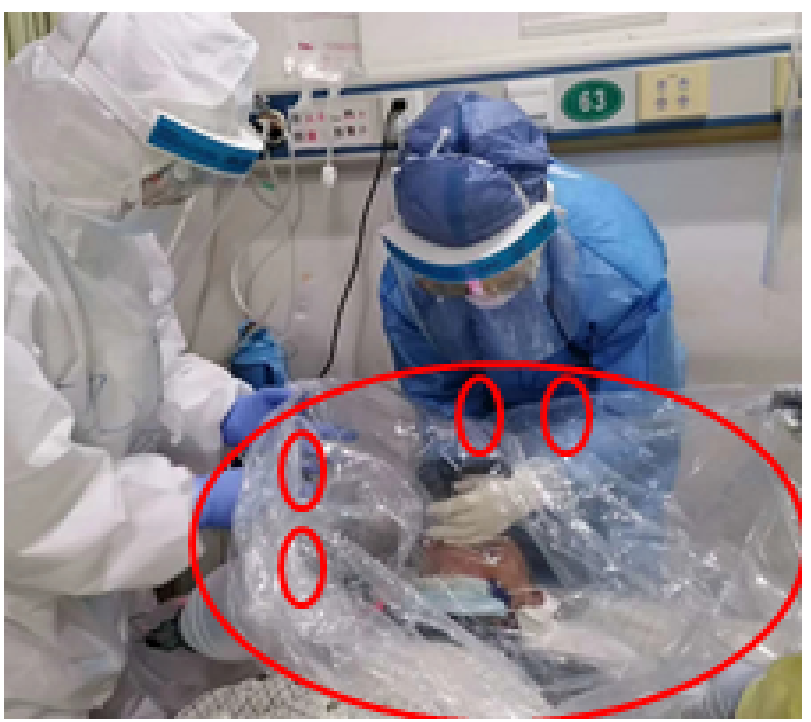

Figure 6: A marked red circle around the peripheral shall be pasted with double-sided adhesive tape to form the relatively closed operation space. 
Improvement measures: Paste the double-sided tape around the edge of the plastic shield for standby, tear the sealing strip when using, and make it closely fit to the upper body and head of the patient, forming a relatively closed space, so as to better prevent the diffusion of the aerosol.

According to the specifications of the plastic shield, if there are two pairs of elastic belts on the top, 4 operation holes can be formed to facilitate the operation under the plastic shield (Figure 6 and Figure 7).

Further improvement measures: By using the protective shield mentioned above, and a continuous suction pipe beside to keep the overall confined space in a relatively negative pressure state, this can effectively reduce the possibility of aerosol spreading outwards (Figure 8).

It can be used for:

Patients who need mask pressure oxygen;

Patients requiring conscious intubation;

Patients who need tracheotomy.

\section{Protective measures for susceptible persons}

(1) The application of three-level protective mea-

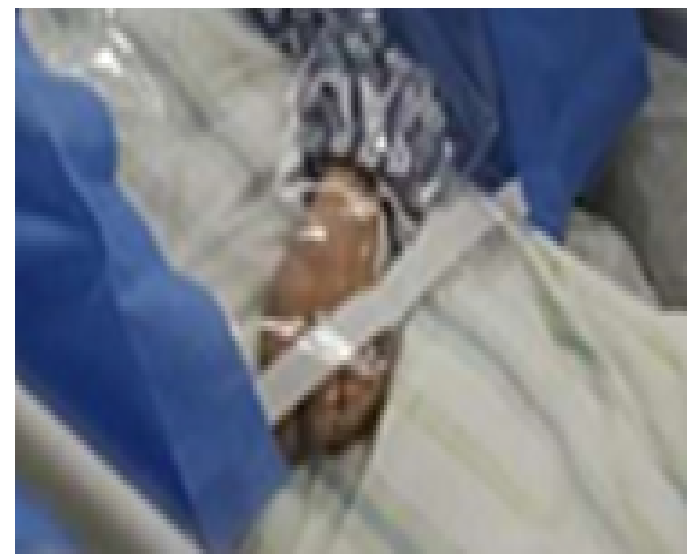

Figure 7: Four small red circles marked inside, are made into operation holes with tightness.

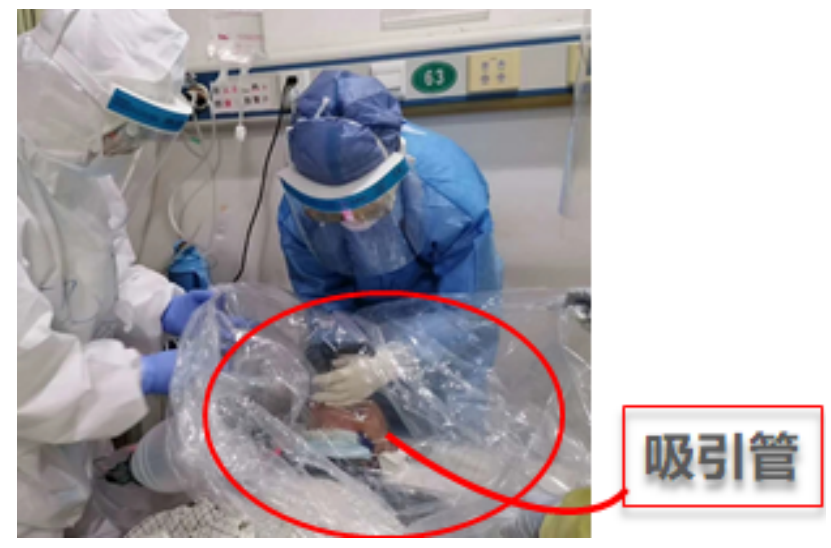

Figure 8: Suction pipe instantly remove the aerosol from the surrounding of the mask. sures

Protective clothing, isolation clothing, work cap, three-layer gloves, protective glasses, N95 mask, isolation shoes and shoe covers (Figure 9).

(2) Strictly implement the dressing process of high risk exposure protective clothing and the removal process of high risk exposure protective clothing, and complete it under the guidance and supervision of professionals if necessary.

(3) In addition to the three-level protection, for the need for special clinical operations, the susceptible (medical staff) should strengthen their own protection. Such as screen, self-made head cover and positive pressure head shield (Figure 10 and Figure 11).

\section{Results}

\section{Based on research results, recommended mea- sures are as follows}

For those with positive pressure head shield:

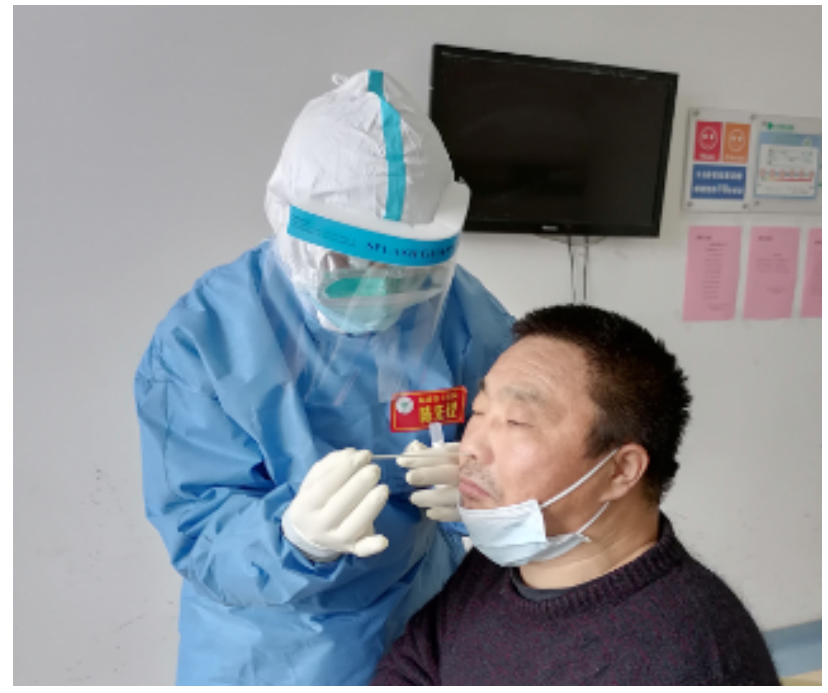

Figure 9: The application of three-level protective measures.

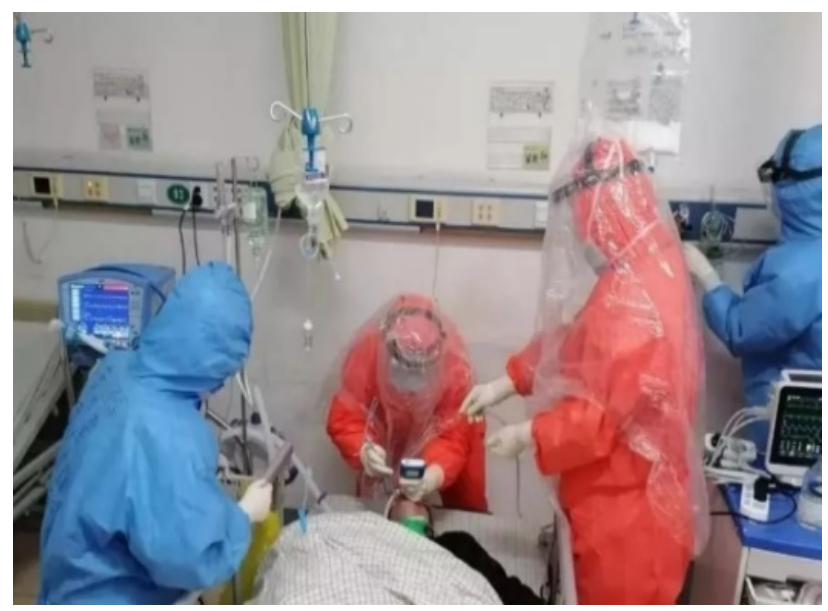

Figure 10: Plastic head shield made by Fujian Union Hospital. 


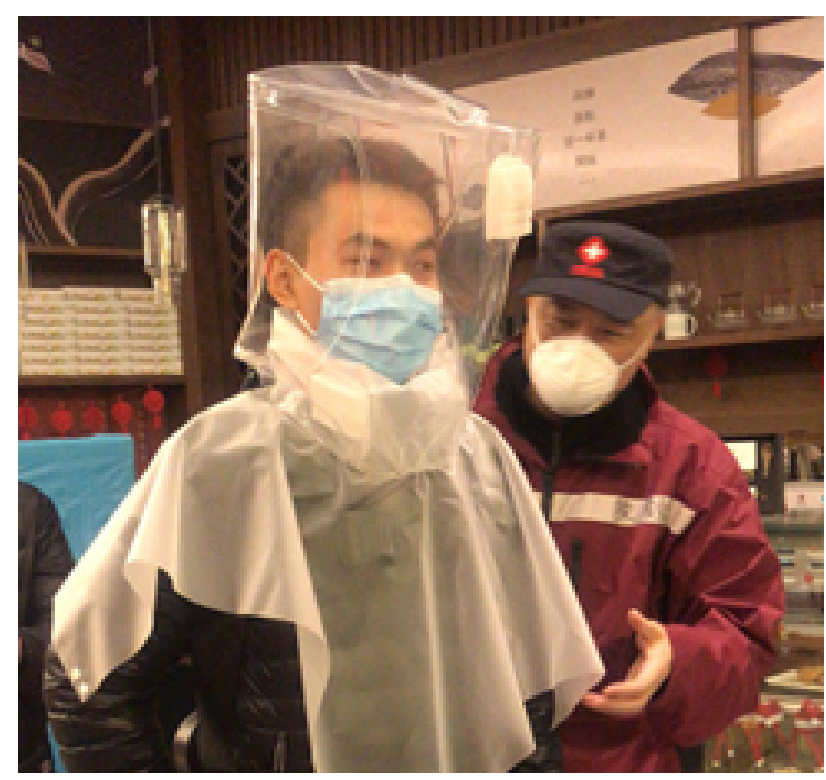

Figure 11: Positive pressure head shield from another medical team.

(1) Personal three-level protection + positive pressure head shield

(2) Personal three-level protection + positive pressure head shield + multi-layer gauze damped with alcohol and covered beyond the mask

(3) Personal three-level protection + positive pressure head shield + transparent plastic shield to form a closed negative pressure space

(4) Personal three-level protection + positive pressure head shield + multi-layer gauze damped with alcohol and covered beyond the mask + transparent plastic shield to form a closed negative pressure space

\section{For those without positive head shield:}

(1) Personal three-level protection + face shield + multi-layer gauze damped with alcohol and covered beyond the mask

(2) Personal three-level protection + transparent plastic shield to form a closed negative pressure space

(3) Personal three-level protection + multi-layer gauze damped with alcohol and covered beyond the mask + transparent plastic shield to form a closed negative pressure space

\section{Discussion}

The emergence of the alternatives has its inevitability. The main reason is that the standard protective articles are expensive and scarce, the wearing process is tedious and time-consuming, and the operator's proficiency is required to be high. In an emergency, the rescue opportunity may be delayed. We analyzed and compared the advantages and disadvantages and reality of different protective interventions during the high exposure risk procedure.

Based on the advantages of different protection models, we propose several improvement schemes which are easy to obtain and very practical. It can provide alternative temporary protection when there is unavailable [5] of advanced equipment such as positive pressure head shield. Limited protection can be provided in the implementation of rapid tracheal intubation or in the protection of infectious diseases transmitted by respiratory system.

Restricted by limited conditions, the safety and effectiveness of the protection has not been confirmed by etiology. However, these measures are simple and easy to use, and the equipment consumables are easy to obtain. As an alternative, to some extent, it has unique value while improving the safety of medical work.

\section{Conclusion}

For patients infected with novel coronavirus and presented severe respiratory syndrome, especially when tracheal intubation were necessary, our study has enumerated detailed solution and examples under certain circumstances, they are different protective measures to reduce the highly exposure risk. In the absence of positive pressure head shield, the on hand material can be taken for proper reconstruction. It will be safer when one or more protective measures were applied in combined. Above all, personal three-level protective measures should be carried out strictly. For those without difficult airway, can receive proper sedation and sufficient muscle relaxation, to provide ideally condition for tracheal intubation, to eliminate the cough reflex of patients and reduce the exposure risk for clinical physicians.

\section{References}

1. Li Q, Guan X, Wu P, Wang X, Zhou L, et al. (2020) Early transmission dynamics in wuhan, China, of novel coronavirus-infected pneumonia. N Engl J Med 382: 1199-1207.

2. Rubin EJ, Baden LR, Morrissey S (2020) Audio interview: Practical measures to help prevent Covid-19. N Engl J Med 382: e32.

3. Ranney ML, Griffeth V, Jha AK (2020) Critical supply shortages - The need for ventilators and personal protective equipment during the Covid-19 Pandemic. N Engl J Med.

4. Truog RD, Mitchell C, Daley GQ (2020) The toughest triage - Allocating ventilators in a pandemic. N Engl J Med.

5. Emanuel EJ, Persad G, Upshur R, Thome B, Parker M, et al. (2020) Fair allocation of scarce medical resources in the time of Covid-19. N Engl J Med.

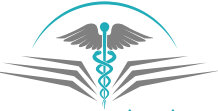

